\title{
A lepto-hadronic model for the high energy emission from the jets of FR I radiogalaxies
}

\author{
Matías M. Reynoso ${ }^{1}$, María C. Medina $^{2}$, and Gustavo E. Romero ${ }^{3}$ \\ ${ }^{1}$ Instituto de Investigaciones Físicas de Mar del Plata \\ (Universidad Nacional de Mar del Plata - CONICET), Mar del Plata, Argentina \\ email: mreynoso@mdp.edu.ar
}
${ }^{2}$ Institute de Recherche sur les Lois Foundamentales de l'Univers (IRFU), Service de Physique de Particules, Commisariat l'Energie Atomique, Saclay, France email: clementina.medina@cea.fr
${ }^{3}$ Instituto Argentino de Radioastronomía (CCT La Plata-CONICET), Villa Elisa, Argentina email: romero@f caglp.unlp.edu.ar

\begin{abstract}
We present a lepto-hadronic model for the VHE emission from the relativistic jets of FR I radiogalaxies. We assume that protons and electrons are accelerated in a compact region near the base of the jet, and they cool emitting multiwavelength radiation as they propagate along the jet. The particle distributions are obtained using an inhomogeneous steady-state transport equation that accounts for the cooling processes as well as the convection of particles in the jet. The dominant processes that contribute to the photon SED are electron and proton synchrotron radiation, inverse Compton interactions, and the inelastic collisions $p p$ and $p \gamma$. The accompanying neutrino output is obtained and the possibility of detection with Km3Net and IceCube is discussed for the cases of Cen A and M87.
\end{abstract}

Keywords. galaxies: active, radiation mechanisms: nonthermal, gamma rays: theory, neutrinos

Outline of the model. FR I radiogalaxies are the AGNs in which the jet makes a large angle with the line of sight. In this work we present a model based of relativistic leptons and hadrons to explain the high energy emission from the jets of these objects. We show the results obtained for the radiogalaxies Cen A and M87, which have been detected by HESS and Fermi. We now enumerate the assumptions made to develop the present model. Matter is captured by the central black hole though a dissipationless accretion disk Bogovalov \& Kelner (2010). A fraction of this accreted material is expelled in two oppositely directed jets. Equipartition between jet kinetic energy and magnetic energy takes place at $z_{0}=50 R_{g}$ from the black hole. The jet has a small half-opening angle $\xi$, a mildly relativistic bulk Lorentz factor $\Gamma_{\mathrm{j}}$ at $z_{0} \&$, and a middle viewing angle $i_{\mathrm{j}}$. The bulk kinetic power of the jet at $z_{0}$ is a fraction of the Eddington power: $L_{\mathrm{j}}^{(\mathrm{kin})}=0.5 q_{\mathrm{j}} L_{\mathrm{Edd}}$. The magnetic field varies along the jet according to $B(z)=B_{0}\left(\frac{z_{0}}{z}\right)^{m}$ with $m \in(1,2)$. $\Gamma_{\mathrm{j}}$ increases slowly as magnetic energy decreases along the jet. Acceleration of relativistic protons and electrons takes place at $z_{\text {acc }}>z_{0}$, where the magnetic energy is in subpartition with the kinetic energy. The power carried by primary relativistic particles, $L_{\mathrm{rel}}=L_{p}+L_{e}$, is a fraction of the bulk kinetic power of the jet, $L_{\mathrm{rel}}=q_{\mathrm{rel}} L_{\mathrm{j}}^{(\mathrm{kin})}$. The relation between the proton and electron power is given by the parameter $a: L_{p}=a L_{e}$.

Method of calculation. The maximum energies are obtained from the balance of the energy losses and gains. The main cooling for electrons is synchrotron radiation, and for protons we consider: $p \gamma$ collisions, synchrotron emission, adiabatic cooling, and $p p$ collisions. Synchrotron photons emitted by electrons, are the main target for IC and $p \gamma$ interactions. They also cause internal absorption of gamma-rays. External absorption by the surrounding gas and dust is taken into account with an optical depth $\tau_{\gamma H}\left(E_{\gamma}\right)=N_{H} \sigma_{\gamma H}\left(E_{\gamma}\right)$. 
Table 1. Parameters used in this work.

\begin{tabular}{|c|c|c|}
\hline Parameter & Cen A & M87 \\
\hline $\begin{array}{c}M_{\mathrm{bh}}: \text { Black Hole mass } \\
L_{\mathrm{j}}^{(\mathrm{kin})}: \text { jet power } \\
\Gamma_{\mathrm{j}}: \text { jet's bulk Lorentz factor at } z_{0} \\
\xi: \text { jet's opening angle } \\
i_{\mathrm{j}}: \text { viewing angle } \\
q_{\mathrm{rel}}: \text { jet's content of relativistic particles } \\
a: \text { hadron-to-lepton ratio } \\
m: \text { magnetic field dependance with z } \\
z_{0}: \text { jet's launching point } \\
z_{\mathrm{acc}}: \text { position of acceleration zone } \\
\Delta z_{\mathrm{acc}}: \text { extent of injection zone } \\
\eta: \text { acceleration efficiency } \\
s: \text { spectral index of injection } Q \\
E_{m i n, p}: \text { minimum energy protons } \\
E_{m i n, e}: \text { minimum energy electrons } \\
N_{H}: \text { gas and dust column density }\end{array}$ & \begin{tabular}{|c}
$10^{8} M_{\odot}$ \\
$6 \times 10^{44} \mathrm{erg} \mathrm{s}^{-1}$ \\
2.5 \\
$5^{\circ}$ \\
$25^{\circ}$ \\
0.1 \\
1 \\
1.5 \\
$7,4 \times 10^{14} \mathrm{~cm}$ \\
$167 R_{g}$ \\
$z_{\mathrm{acc}} \tan ^{-2}$ \\
$10^{-2}$ \\
1.8 \\
$3.5 \mathrm{GeV}$ \\
$51 \mathrm{MeV}^{23} \mathrm{~cm}^{-2}$
\end{tabular} & $\begin{array}{c}6 \times 10^{9} M_{\odot} \\
6.7 \times 10^{46} \mathrm{erg} \mathrm{s}^{-1} \\
3.5 \\
1.5^{\circ} \\
20^{\circ} \\
0.1 \\
175 \\
1.5 \\
4.4 \times 10^{16} \mathrm{~cm} \\
200 R_{g} \\
z_{\mathrm{acc}} \tan ^{-4} \\
10^{-4} \\
2.4 \\
4.5 \mathrm{GeV} \\
77 \mathrm{MeV}^{20} \mathrm{~cm}^{-2}\end{array}$ \\
\hline
\end{tabular}
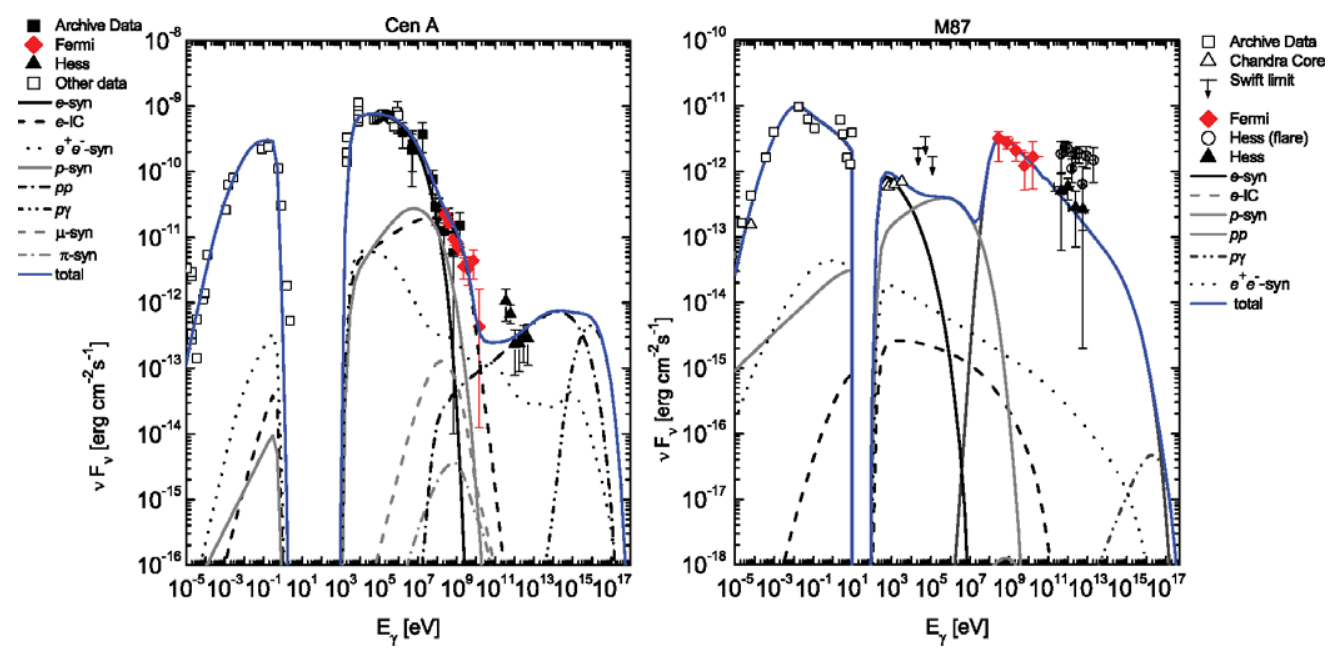

Figure 1. SEDs obtained for Cen A and M87.

The cross section $\sigma_{\gamma H}\left(E_{\gamma}\right)$ is taken as in Ryter (1996). The distribution of particles are obtained solving a steady-state transport equation with cooling and convection:

$$
v(z) \frac{\partial N(E, z)}{\partial z}+\frac{\partial b(E, z) N(E, z)}{\partial E}+\frac{N(E, z)}{T_{\mathrm{dec}}(E)}=Q(E, z),
$$

where $b(E, z)=-\frac{d E}{d t}$. The production of secondary particles $\left(\pi, \mu, e^{ \pm}, \nu_{i}\right)$ in the jet by $p \gamma, p p$, and $\gamma \gamma$ interactions is taken into account.

Results for Cen $A$ and $M 8 \%$. Table 1 presents the set of parameters used for applying our model to Cen A and M87. The resulting SED is shown in Fig 1. The computed SEDs are basically consistent with the multiwavelength data for both sources. The main processes are synchrotron emission of electrons and protons, IC interactions, $p p$ and $p \gamma$ collisions. The accompanying neutrino output obtained is found to be stronger for Cen than for M87 for energies above $1 \mathrm{TeV}$ (see Reynoso et al. (2010) for details).

\section{References}

Bogovalov, S. V. \& Kelner, S. R. 2010, IJMPD, 19, 339.

Morganti, R., Oosterloo, T., Struve, C., \& Saripalli, L. A\& A, 485, L5.

Reynoso, M. M., Medina, M. C., \& Romero, G. E. 2010, submitted, arXiv:1005.3025.

Ryter, C. E., 1996 ApSS, 236, 285. 